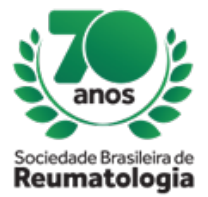

\title{
T-CELL LARGE GRANULAR LYMPHOCYTE LEUKEMIA: A CASE REPORT
}

Sandra Helena Machado (Hospital de Clinicas de Porto Alegre, Porto Alegre, RS, Brasil), Yasminne Rocha (UFRGS/ HCPA, Porto Alegre, RS, Brasil), Fernanda Becker Tabajara (Hospital de Clinicas de Porto Alegre, Porto Alegre, RS, Brasil), João Victor de Andrade Aguas (UFRGS/HCPA, Porto Alegre, RS, Brasil), Leticia Rossetto Daudt (UFRGS/HCPA, Porto Alegre, RS, Brasil), Zingara dos Santos Alves (HCPA/UFRGS, Porto Alegre, RS, Brasil), Bruno Batista Silva (HCPA/UFRGS, Porto Alegre, RS, Brasil)

\section{BACKGROUND}

T-cell large granular lymphocyte (T-LGL) leukemia is a clonal proliferation of cytotoxic T cells, characterized by peripheral blood and marrow lymphocytic infiltration with LGL. Common clinical manifestations are splenomegaly, cytopenias (neutropenia), anemia, and thrombocytopenia. This condition is often associated with autoimmune disorders and other lymphoproliferative disorders. T-LGL leukemia has been rarely reported in children. We report a child with T-LGL leukemia who presented with neutropenia and went on to develop juvenile systemic lupus erythematosus

\section{CASE REPORT}

Female infant with five years old, was admitted to critical care unit with severe refractory septic shock and pancytopenia, important colitis in the cecum and ileo-cecal region, and anterior and posterior anal laceration .Colonoscopy showed edemaciated ileo-cecal valve, mucosa of the cecum presenting three deep ulcers, three lacerations in the anus. Cecal biopsy showed chronic colitis and cryptitis and mild cryptic microabscesses. Based on these results, lesions in cecum were tought to be the result of an infectious process not seen at the time of initial presentation, and now in remission. Due to 3 episodes of fever during hospitalization, complementary tests were ordered, reporting leucopenia with neutropenia in 2 occasions, which led to the investigation of cyclic neutropenia or autoimmune neutropenia. Bone marrow biopsy was performed, and the medulogram showed discrete hypocellularity, normomaturative, and with normal morphology. Immunophenotyping was unchanged. Infant also had positive rheumatologic markers (FAN and antilupus coagulant) but no signs of systemic autoimmune disease.

She presented febrile neutropenia again, associated with diarrhea, and was readmitted for treatment and further investigations of etiology. Chest X-ray and urine tests showed no alterations and abdominal echo had signs of colitis as well as in the first hospitalization. Metronidazole and cefepime were started. Patient evolved with leukopenia improvement, but still with neutropenia. It was decided to repeat the bone marrow biopsy and to carry out a new immunophenotyping., which led to the diagnosis of LGL leukemia.

Methotrexate, prednisolone, filgrastima and sulfamethoxazole-trimethoprim were the drugs of treatment. She presented improvement of the neutropenia, being discharged with outpatient follow-up. Since then, she did not have neutropenia or leukopenia.

\section{CONCLUSION}

Although rare, T-LGL leukemia must be considered in children with pancytopenia and autoimmune disorders, 